\title{
Survey of Musculoskeletal Pain among Dental Clinician in Kulliyyah of Dentistry IIUM
}

\author{
Susi Sukmasari ${ }^{1}$, Ahmad Faisal Ismail ${ }^{1}$, Arbaatul Arroshikin Nazirah Hamshah ${ }^{2}$ \\ ${ }^{1}$ Kulliyyah of Dentistry, International Islamic University Malaysia Kuantan \\ ${ }^{2}$ Kulliyyah of Allied Health Sciences, International Islamic University Malaysia Kuantan
}

\begin{abstract}
This study aimed to determine the prevalent site of musculoskeletal pain and its associated risk factors among dental clinicians at the Kulliyyah of Dentistry, IIUM. A validated, pilot-tested self-administered questionnaires were distributed to 160 dental clinicians between March and June 2014 using convenience sampling method. Inclusion criteria includes dentist, clinical supervisors or clinical dental students. Dental clinicians whom were diagnosed with musculoskeletal disorders were excluded. Data obtained were recorded and analyzed using statistical package for the social science (SPSS) software version 19. Pearson correlation test was used to determine the association between the risk factors and pain sites. A total of 146 participants completed the questionnaire $(91.3 \%$ response rate). The sample consist of 40 dentists $(27.4 \%)$, 36 Year 3 students (24.7\%) and 35 participants (24\%) respectively from Year 4 and Year 5 . There was high prevalence of neck pain $(79.5 \%)$, back shoulder pain $(74.7 \%)$ and lower back pain $(71.9 \%)$ among dental clinicians. Significant correlation was found between physical activities with upper body region pain sites $(r$ $=0.170, p=0.042)$ and lower body region pain sites $(r=0.221, p=0.008)$. There was a significant association between repetitive movements and wrist bending with upper and lower body region pain sites $(r=0.320, p=$ $<0.001)(r=0.278, p=0.001)$. No correlation between awkward postures with upper and lower body pain sites. This paper will relate to the Islamic teaching on moderation in working and in maintaining healthy lifestyle. The most prevalent site of musculoskeletal pain experienced by dental clinicians was at the neck area and the least was on the left arm. Repetitive movements, wrist bending and physical activities were associated with upper and lower body region pain sites.
\end{abstract}

KEYWORDS: Musculoskeletal pain, risk factors, dentistry.

\section{INTRODUCTION}

Dentistry is a healthcare field that involves long working shift of repetitive movement, different body posture and stress that can contribute significantly to both musculoskeletal disorders (MSDs), psychological stress and fatigue. Dental professionals or oral healthcare providers comprised of dentists, dental assistants, dental hygienists, dental students, dental nurses, dental laboratory workers, dental technicians and dental office workers. ${ }^{1}$ Dental clinicians are prone to occupational health hazards and the development of cumulative trauma disorder. Their working routine requires them to sit or stand for a long period where they need to maintain their head, neck and shoulders in a fixed position. ${ }^{2}$ Prevalence of musculoskeletal disorder is high among dental clinicians, probably because of high physical work load, such as visualization on a narrow working area, repeated unnatural working postures, precise and occasionally forceful hand movements. ${ }^{3,4}$ Other risk factors are

Corresponding author:

Ahmad Faisal Ismail

Kulliyyah of Dentistry,

International Islamic University Malaysia,

Jalan Sultan Ahmad Shah,

25200 Kuantan, Pahang

Email:drfaisal@iium.edu.my the use of vibrating instruments or poor lighting during dental treatment. ${ }^{5}$ The common sites of musculoskeletal discomforts among dental personnel are the neck, shoulder and lower back regions. $^{5}$ Studies indicate that back, neck and shoulder or arm pain were presented in up to $81 \%$ among dental operators. ${ }^{4}$ Some authors suggested that injuries caused by work-related musculoskeletal disorder can be prevented by applying ergonomic dental equipment and instrument design. ${ }^{2}$ All of these factors might impair the working capability of dental clinician and performance of the dental treatment itself. Thus, this study aimed to determine the prevalent site of musculoskeletal pain and the associated risk factors among dental clinicians in the Kulliyyah of Dentistry, International Islamic University Malaysia (IIUM).

\section{MATERIALS AND METHOD}

This study was conducted at the Kulliyyah of Dentistry, International Islamic University Malaysia, Kuantan, Pahang. Upon approval by local ethical committee (Reference IREC 179), a validated, pilottested questionnaire were distributed to 160 dental clinicians between March and June 2014 using convenience sampling method. Sample size (n) was calculated by using Raosoft online sample size calculator. Inclusion criteria includes dentist, clinical supervisor and clinical dental students. 
IMJM Volume 17 Special Issue No 2

Exclusion criteria includes clinicians diagnosed with musculoskeletal disorders. Close-ended questions such as listing questions, Likert scale questions and pain rating scale were used in this questionnaire. All data collected were subjected to statistical test using Statistical Package for Social Sciences (SPSS) version 19. Pearson correlation was used to determine the association between the risk factors and the musculoskeletal pain sites.

\section{RESULTS}

A total of 146 dental clinicians participated in this study ( $91.3 \%$ response rate). The dropped out reason were due to incomplete or missing data and nonresponse. Majority of the respondents were female with 110 participants (75.3\%) and male with 36 participants $(24.7 \%)$. The mean age of participants was 26.90 years $(S D=6.545)$. The mean body mass index (BMI) was calculated at $22.79 \mathrm{~kg} / \mathrm{m}^{2}$ $(\mathrm{SD}=5.759)$. Table 1 shows categorization of respondents based on their group.

Table 1. Category of respondent

Category Number $(n) \quad$ Percentage

\begin{tabular}{lll} 
Dentist / Supervisor & 40 & 27.4 \\
Year 5 student & 35 & 24.0 \\
Year 4 student & 35 & 24.0 \\
Year 3 student & 36 & 24.6 \\
& & \\
TOTAL & 146 & 100 \\
\hline
\end{tabular}

Majority of respondents were right handed (84.6\%), while 13 were left handed $(9.1 \%)$ and nine respondents used both of their hands to perform dental treatment (6.3\%). Majority of the respondents were in straight position when performing dental treatment which comprised of 106 respondents $(72.6 \%)$ while 40 respondents (27.4\%) were in bent position.

Dental procedures such as scaling, polishing, filling, extraction, impression-taking, placement of fixed orthodontic appliance, suction and retraction were classified as repetitive movements and wrist bending procedure. Scaling was the most common dental procedure performed $(n=87,59.6 \%)$, followed by polishing $(n=73,50.0 \%)$, filling $(n=81,55.5 \%)$ and extraction $(n=67,45.9 \%)$. The least common procedure performed was placement of fixed orthodontic appliance procedure $(n=3,0.02 \%)$.

Figure 1 showed the prevalent pain sites for upper body region. Neck pain was the most common site with $79.5 \%$ of respondents $(n=116)$ in contrast to pain on left arm with 67 respondents (45.9\%) which is the least common. Figure 2 showed the prevalent pain sites for lower body region. The most common site for lower body region was right and left foot $(n=77,52.7 \%$ respectively) while the least common was lower leg $(n=66,45.2 \%)$.

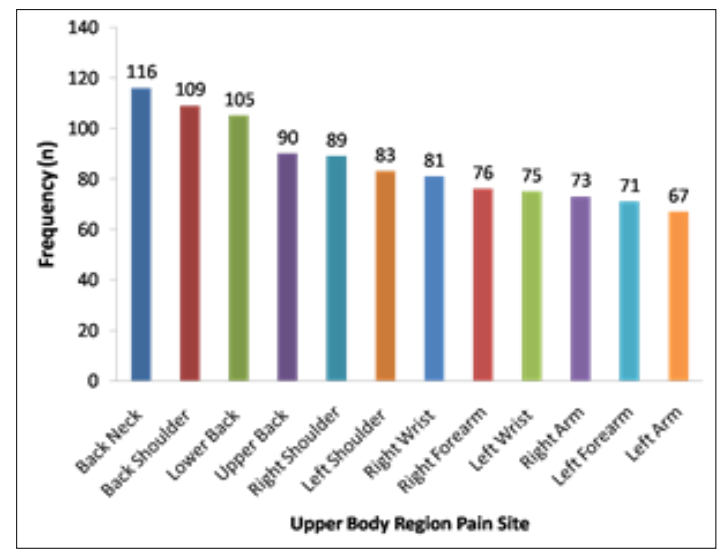

Figure 1. Site for upper body region

There is a significant correlation between physical activities and upper body region pain sites $(r=$ $0.170, p=0.042$ ), specifically between repetitive movements and wrist bending with upper body region pain sites $(r=0.320, p=<0.001)$. However, exposure to hand instruments with upper body region pain site represents no significant correlation $(r=0.111, p=0.184)$. Furthermore, no significant correlation was found between awkward postures and upper body region pain sites $(r=$ 0.059, $p=0.484$ ).

For lower body region pain site, there is significant correlation between physical activities and lower body region pain sites $(r=0.221, p=0.008)$. Significant correlation was also demonstrated between repetitive movements and wrist bending with lower body region pain sites $(r=0.278, p$ $=0.001$ ). However, exposure to hand instruments and awkward postures showed no significant correlation with lower body region pain site $(r=$ $0.077, p=0.357, r=0.024, p=0.781$ respectively).

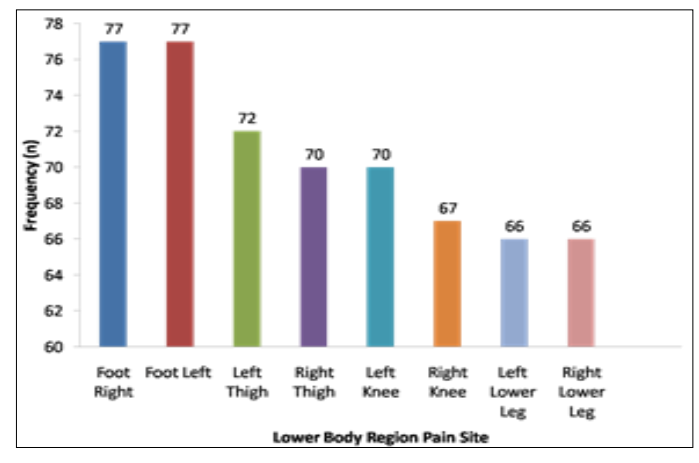

Figure 2. Site for lower body region 


\section{DISCUSSION}

Gender can be considered as a potential risk factor for developing musculoskeletal disorder. This is supported by a study carried out by Dajpratham et al. ${ }^{6}$ which showed that the distribution of cervicobrachial pain was higher in female compared to male respondents. Furthermore, Khan and $\mathrm{Chew}^{2}$ stated that female dental students showed a higher prevalence of work musculoskeletal disorder symptoms than males. However, since the proportions of male students were small in our study, it was not appropriate to determine the differences in musculoskeletal pain prevalence rates by gender. This was due to the fact that the intake of dental students of IIUM were dominated by females. Recently, in some countries, the number of females in the field of dentistry is increasing significantly. ${ }^{7,8}$ Therefore, this study is in line with Smith and Leggat ${ }^{9}$ where they suggested that gender might be a confounding variable rather than a true risk.

Age can be contemplated as a possible factor in developing musculoskeletal disorder symptoms. According to a study done by Rabiei et al. ${ }^{10}$ age as a risk factor was statistically significant associated with occurrence of neck pain, shoulder pain and lower back pain among dentists. The mean age of dental clinician in KOD was 26.9. This matches with the fact that most of the respondents were students from year 3, 4 and 5 . This study can be useful as a precaution for young dentists regarding the possible future diseases.

At the Kulliyyah of Dentistry, IIUM, there are only three orthodontists providing placement of fixed appliances on patients. This explains why the procedure was uncommon in the clinic. Meanwhile, scaling is a procedure which requires dental students and dentists to sit and treat the patient in a prolonged period of time. It involves repetitive movement in removing calculus on the tooth surfaces from the whole mouth. Furthermore, the instrument used can either be a manual or ultrasonic scaler with vibration. This procedure is required on all patients before they were referred to other departments for treatments such as filling, extraction or prosthodontic (denture) appliances.

There was a significant correlation between repetitive movements and wrist bending with both upper and lower body region pain sites. This can be explained in a condition where the median nerve may become compressed within the carpal tunnel and hence leading to Carpal Tunnel Syndrome (CTS). On top of that, repetitive force and motion to the wrist will cause an inflammation and swelling of the tendons within the carpal tunnel, and repeated wrist flexion and hyperextension also might cause the tendon to swell. This can be supported by a study done by Madaan and Chaudhari ${ }^{4}$ which stated that scaling and polishing procedure in Periodontics field was the highest clinical activity that produced musculoskeletal discomfort among the dental students. Since most of our respondents were right- handed, they repeatedly assume the opposite position rotating the neck to the left with sidebending to the right to gain better visibility. ${ }^{11}$

Grasping small instruments and vibrating hand tools such as slow-speed hand piece and ultrasonic scaler for a prolong period of time are risk factors that might initiate CTS. This is because, grasping or pinching an object for a long period might reduce the blood flow and cause inflammation of tendons, also known as a tendonitis. Most of the participants in this study experienced pain at both right and left wrists. However, there was no significant correlation found between grasping small instruments and using vibrating hand tools with both upper and lower body region pain site. Khan and $\mathrm{Chew}^{2}$ highlighted that hand and finger discomfort were significantly correlated with the number of hours using vibrating instruments. In addition to this, there was a statistically significant correlation between exposure to vibrating tools and shoulder pain among dentists. ${ }^{12}$

Most of ergonomic injuries in dentistry resulted from counteracting rotational force or torque for prolonged period of time. It occurs when a system becomes unbalanced and relates to operator position is their own head. As an effort to achieve a direct vision and maintain focus into the oral cavity, dentists need to lean or tilt forward. ${ }^{13}$ Furthermore, forwarding head postures may precede the syndrome, precipitating muscle imbalances, ischemia, trigger points, or cervical disc degeneration or herniation. The dentist lean type is described as head declination with the fulcrum point positioned in the cervical area of the neck. In this study, the result showed that most of the operator's back position were straight, and as an effort to obtain a direct vision, they forwarded the head with the fulcrum in the neck. In the erect sitting position, the center of gravity is forward of the ischia. With the lordosis lumbar slightly flattened, it may transmit about $25 \%$ of body weight to the floor through the lower extremities. However, in the slouched sitting position, the center of gravity is posterior to the ischia, the lumbar lordosis is reversed, and far less body weight is transferred to the floor via the lower extremities. ${ }^{14}$

Preventing chronic pain in dentistry may require a paradigm shift within the profession regarding clinical work habits, including proper use of ergonomic equipment, frequent short stretch breaks and regular strengthening exercise. Prophet Muhammad advised his followers, to work, to be energetic, and to start their day early, all of which are conditions for a healthy body. He said "O God, make the early morning hours blessed for my nation" (Imam Ahmad). Another reason why people of faith make effort in this regard is because physical health is a quality that Allah stressed on in the Qur'an. This paper also relates to Islamic teaching on moderation in working and to maintain a healthy lifestyle. The balance between working abilities and healthy well-being was mentioned in 
the Quran (2:143) "And we have made you a median community / a people of moderation / a balanced medium in order that you may be a testimony/model for humanity".

Our study act as a platform for the importance of musculoskeletal pain in dentistry. This can serve as a baseline data for future research to evaluate the working environment, such as rearrangement of equipment and instruments, treatment procedures and stretching exercises during working hours.

\section{CONCLUSION}

Our study concluded that the most prevalent site of musculoskeletal pain experienced by dental clinician was neck pain and the least common was left arm. Repetitive movements, wrist bending and physical activities were associated with upper and lower body region pain sites.

\section{REFERENCES}

1. Kumar SP, Kumar V, Baliga M .Work-related musculoskeletal disorders among dental professionals: An evidence-based update. Indian Journal of Dental Education. 2012; 5:1-8

2. Khan SA, Chew KY. Effect of working characteristics and taught ergonomics on the prevalence of musculoskeletal disorders amongst dental students. BMC Musculoskeletal Disorder. 2013; 14:118

3. Abduljabbar TA. Musculoskeletal Disorders among Dentists in Saudi Arabia. Pakistan Oral Dental Journal. 2008; 28:135-144

4. Madaan V, Chaudhari A. Prevalence and Risk Factor associated with Musculoskeletal Pain among Students of MGM Dental College: A Crosssectional Survey. Journal of Contemporary Dentistry. 2012; 2:22-27

5. Sankar SG, Reddy PV, Reddy BR, Vanaja K. The prevalence of work-related musculoskeletal disorders among Indian orthodontists. $J$ Ind Orthod Soc. 2012; 46:264-268

6. Dajpratham P, Ploypetch T, Kiattavorncharoen $\mathrm{S}$, Boonsiriseth K. Prevalence and Associated Factors of Musculoskeletal Pain among the Dental Personnel in a Dental School. J Med Assoc Thai. 2010; 93:714-721

7. Pallavi SK, Rajkumar GC. Professional practice among woman dentist. J Int Soc Prev Community Dent. 2011; 1:14-19.

8. McKay J. The feminization of dentistry: Implications for the profession. J Can Dent Assoc. 2012; 78:c1.

9. Leggat PA, Kedjarune U, Smith DR. Occupational Health Problems in Modern Dentistry: A Review. Ind Health. 2007; 45:611-621.

10. Rabiei M, Shakiba M, Shahreza HD, Talebzadeh M. Musculoskeletal Disorders in Dentists. International Journal Of Occupational Hygiene. 2012; 4:36-40.

11. Valachi B, Valachi K. Mechanisms leading to musculoskeletal disorders in dentistry. J Am Dent Assoc. 2003; 134:1344-1350.

12. Alexopoulos EC, Stathi IC, Charizani F. Prevalence of musculoskeletal disorders in dentists. BMC Musculoskeletal Disorder. 2004; 5:16.

13. Carter J. The inevitability of neck and back pain. In: Dentistry IQ [online]. Available at: www.dentistryiq.com, 2007.

14. Schafer RC. Clinical biomechanics: musculoskeletal actions and reactions. $2^{\text {nd }}$ ed. London: Williams \& Wilkins, 1987 